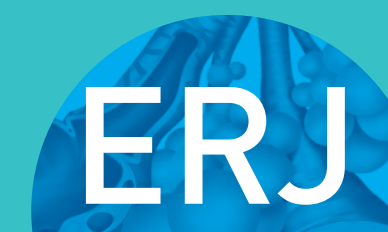

open research
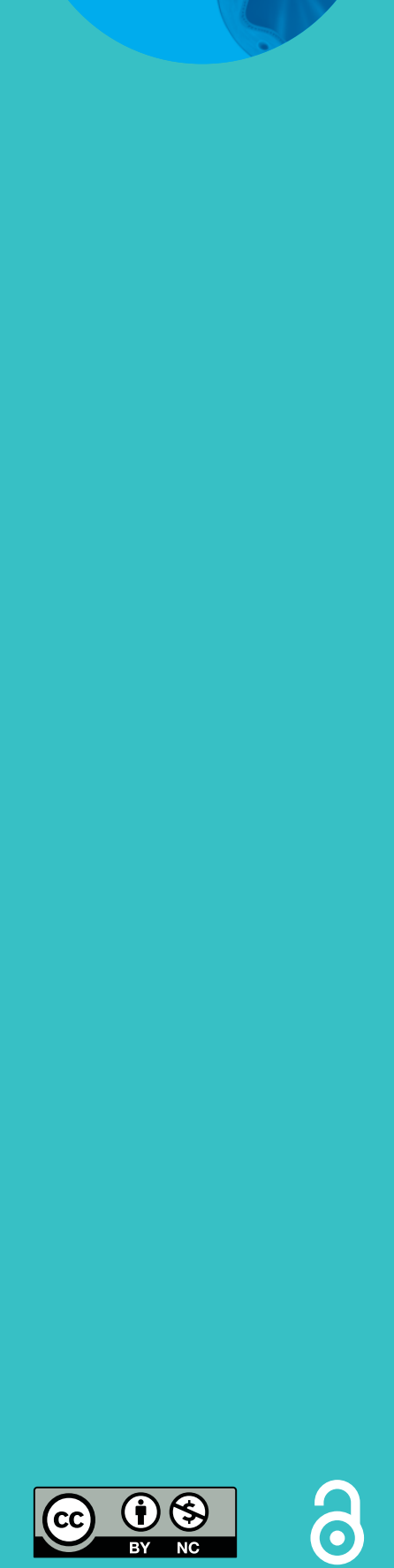

\section{Fungal contamination of the respiratory tract and associated respiratory impairment among sawmill workers in India}

\author{
Asit Adhikari ${ }^{1}$, Subhashis Sahu ${ }^{1}$, Arghya Bandyopadhyay ${ }^{2}$, Paul D. Blanc ${ }^{3}$ and \\ Subhabrata Moitra ${ }^{4}$
}

Affiliations: ${ }^{1}$ Ergonomics and Occupational Physiology Laboratory, Department of Physiology, University of Kalyani, Kalyani, India. ${ }^{2}$ Department of Chemical Sciences, Indian Institute of Science Education and Research, Kolkata, India. ${ }^{3}$ Division of Occupational and Environmental Medicine, University of California San Francisco, San Francisco, CA, USA. "Molecular Respiratory Research Laboratory, Chest Research Foundation, Pune, India.

Correspondence: Subhabrata Moitra, Dept of Respiratory Medicine and Allergology, BMC, Lund University, Lund- 22184, Sweden. E-mail: subhabrata2207@gmail.com

ABSTRACT Wood processing workers are exposed to wood-associated microbiological contaminants, including fungi. Our aim was to study the potential association between sputum fungus and adverse respiratory effects in such workers.

In a group of sawmill workers, we administered a respiratory questionnaire, performed lung function testing and quantified the proportions of leukocytes in spontaneously expectorated sputum samples. We identified fungal species by DNA sequencing.

Of 54 sawmill workers, 19 yielded fungal positive sputum samples (mean age $42.5 \pm 10.4$ years) and 35 were negative for fungus (mean age $36.9 \pm 5.2$ years). The fungus was identified as Candida sp. in all samples. Those with fungal-positive sputum, compared to others, reported more cough (26\% versus 63\%) and haemoptysis $(6 \%$ versus $37 \%$ ) (both $\mathrm{p}<0.05)$, manifested reduced forced midexpiratory flow rates (FEF25-75\%) (82.3 \pm 4.5 versus $69.2 \pm 9.9 \%$ predicted, $\mathrm{p}<0.001)$, and had higher sputum eosinophil counts (median 9.25 versus $3.25 \%, \mathrm{p}<0.01$ ). Reduction of $\mathrm{FEF} 25-75 \%$ was associated both with fungus detection in sputum $(-12.7 \%, 95 \%$ CI $-8.5--16.9 \%)$ and sputum eosinophils $(-2.1 \%$ per $1 \%$ increase in eosinophils, 95\% CI $-1.5--2.8 \%$ ) (both $\mathrm{p}<0.001$ ).

In sawmill workers, Candida sp. detectable in sputum was associated with respiratory symptoms, sputum eosinophilia and reduced $\mathrm{FEF} 25-75 \%$.

@ERSpublications

Candida sp. contamination in the airways is associated with adverse respiratory effects in sawmill workers http://ow.ly/RHAqe

Received: May 052015 | Accepted after revision: Aug 282015

Conflict of interest: None declared.

Copyright $\odot$ ERS 2015. This article is open access and distributed under the terms of the Creative Commons Attribution Non-Commercial Licence 4.0. 


\section{Introduction}

Lumber and wood processing and manufacturing is a common industry worldwide. Wood dust, a major byproduct of such production, has been associated with a range of adverse respiratory effects among those occupationally exposed in milling, carpentry, cabinet making and a number of other related trades. Adverse effects include wood dust exposure-related respiratory tract symptoms, airflow limitation and bronchial hyperresponsiveness [1-10].

Aside from the adverse effects of the wood itself, microbial contamination has also been shown to be a major exposure factor in this industry, particularly in the initial phases of lumber milling. Exposures of concern include both bacteria and fungi, with airborne endotoxin presumed to be a major mediator of at least some wood dust-associated adverse effects [6,11-13]. Multiple investigators have quantified the load of airborne microorganisms in the working environment of wood processing [6, 14-21].

Although bacteria and bacterially derived endotoxins have been a dominant hygiene concern in this industry, a number of investigators have shown that fungal contamination also contributes to respiratory symptoms, lung function impairment and airway hyperresponsiveness among wood processing workers [1, 13, 18, 20-23]. Despite the potentially important role of fungal contamination of wood dust, however, the presence of fungal spores in the sputum of exposed wood workers has not been assessed, either as a measure of exposure or as a potential correlate of adverse effects of this working environment.

We became interested in possible fungal exposure in wood mill workers when a preliminary study of sputum cell viability from one such facility demonstrated the presence, in multiple samples, of what appeared to be yeast. We hypothesised that the presence of fungus in sputum samples would serve as a biomarker of exposure to sawmill dust potentially associated with symptoms and lung function impairment. To test this hypothesis, we studied a group of wood industry workers heavily exposed to dust among whom we systematically evaluated respiratory health and characterised the fungal species, if any, in their sputum samples.

\section{Methods}

\section{Study design and study population}

The wood processing facilities studied were all primary mills where logs were stripped, trimmed and sawn into lumber boards primarily used for furniture making. Prior to milling, the logs are stored in an outdoor holding area that is subject to ambient environmental conditions, including drenching precipitation during the rainy season. The major species processed were predominantly hardwoods such as margosa (Azadirachta indica), mango (Mangifera indica), sal (Shorea robusta), teak (Tectona grandis) and mahogany (Toona ciliata). The four wood mills were chosen as a convenience sample based on their proximity to the laboratory at which the sputum the samples were analysed.

We carried out this study in Kalyani (population 450 000) in West Bengal, India. We identified four small wood working mills employing, in total, 79 workers. We attempted to recruit the entire workforce. The final study group included those who consented to participation, completed the study question, performed adequate spirometry and were able to produce an adequate sample of expectorated sputum for analysis.

Although airborne dust sampling was not carried out to quantify exposure levels, grossly, the work process was extremely dusty, generating visible piles of settled saw dust several feet high near the production lines. There were no specific dust suction collection systems in place at any of the sites and nothing beyond makeshift personal respiratory protection (e.g. mouth-covering scarves) was employed. The study was carried out over a 2-3-week period in the dry season. Lung function studies and sputum collections were carried out on site at each mill. Routine work operations continued during study times. The study was approved by the Departmental Research Committee of the Dept of Physiology, University of Kalyani, and we obtained signed informed consent from the participants before participation.

\section{Respiratory questionnaire}

We used an English-Bengali back-translated version of European Community Respiratory Health Survey (ECRHS-II) questionnaire to inquire about respiratory health, occupational exposure and lifestyle factors of the participants. Although the translated version of this questionnaire has not been validated by formal assessment, we have employed this questionnaire in our previous studies [24-26]. Subjective respiratory complaints in the previous 12 months included: 1) acute or chronic wheezing or whistling of the chest; 2) production of phlegm; 3) acute or chronic cough; 4) breathing trouble; 5) appearance of blood in sputum (haemoptysis); and 6) skin symptoms such as dryness, rash or irritation.

\section{Lung function testing}

We measured lung function using a computerised spirometer (Maestros Mediline Systems Limited, Mumbai, India) according to the American Thoracic Society (ATS)/European Respiratory Society (ERS) 
guidelines for spirometry [27]. We calibrated the spirometer prior each testing day with a 3-L fixed-volume calibration syringe. We calculated the predicted values of lung function variables using prediction equations for the Indian population [28]. The quality of each spirogram was evaluated for acceptability and repeatability in accordance with the ATS/ERS criteria [27]. To achieve accurate and replicable spirograms, each participant completed between three and eight spirometry manoeuvres. Spirometry was performed at a distance sufficiently removed from the mills to avoid any marked interference from workplace contaminants.

\section{Assessment of sputum}

We collected spontaneously expectorated sputum samples by asking each participant to expectorate into a sterile polypropylene tube (Tarsons Ltd, Kolkata, India). Sputum samples were processed using the method of Popov et al. [29] as modified and previously reported in detail elsewhere [30]. In brief, the sputum samples were poured into a Petri dish and all visible white cloudlets with no squamous cell contamination were carefully picked out and processed with freshly prepared $0.1 \%$ dithiothreitol (DTT) (Sisco Research Laboratories, Mumbai, India). The suspension was filtered through a $25-\mu \mathrm{m}$ nylon filter, as described previously [30], to remove all squamous or epithelial cell contamination. Cell viability was assessed by trypan blue staining. Cytospin slides were prepared and Leishman stained according to the method of SARAIVA-Romanholo et al. [31]. We performed differential cell counts of the sputum samples after achieving $\geqslant 80 \%$ intact nonsquamous cells, irrespective of the degree of cell viability or squamous cell contamination during viability determination.

\section{Fungal characterisation}

In order to characterise fungal contamination of the expectorated sputum, an aliquot of each crude sputum sample (without adding DTT) was placed in a clean polypropylene tube and mixed with PBS ( $\mathrm{pH}$ 7.4). The ratio of PBS to sputum was kept at $1: 1$. A $100-\mu \mathrm{L}$ aliquot of thoroughly mixed sputum sample was incubated in potato dextrose agar medium for $24 \mathrm{~h}$ at $37^{\circ} \mathrm{C}$. All the fungal colonies on the agar plates were similar in morphology; one of the colonies was selected from the plate and grown in potato dextrose liquid broth under the same incubation conditions as noted above. We also collected saliva as a control from each of the workers to test for the presence of fungus in the oral cavity that might account for the sputum finding. We collected the saliva samples from the cheek, gum and sublingual areas using a sterile cotton swab (Himedia, Mumbai, India) prior to sputum expectoration. We directly examined sputum samples for the presence of fungus and cultured these samples using the same methods as for sputum.

To confirm whether the colonies represented homogenous species, we sequenced additional colonies from the agar plates randomly. For identification of the isolated culture, sequencing of the conserved genomic region was carried out using standard primer pair for the amplification (sequencing service was provided by Amnion Biosciences Pvt Ltd, Bengaluru, India). The amplicon used for the identification was $18 \mathrm{~S}$ ribosomal RNA gene, partial sequence; internal transcribed spacer 1 and 5.8S ribosomal RNA gene, complete sequence; and internal transcribed spacer 2, partial sequence. The 488-base pair amplicon sequence was then searched for the similarity in US National Center for Biotechnology Information (NCBI) nucleotide collection database using the Nucleotide Basic Local Alignment Search Tool (BLASTN) using standard search parameters [32]. A phylogenetic tree was constructed later using first 10 hits from NCBI BLASTN search results and the query sequence using Molecular Evolutionary Genetics Analysis version 4.1 [33]. The evolutionary history was inferred using the neighbour-joining method. The bootstrap consensus tree inferred from 500 replicates was taken to represent the evolutionary history of the taxa analysed. Branches corresponding to partitions reproduced in $<50 \%$ bootstrap replicates were collapsed. The percentage of replicate trees in which the associated taxa clustered together in the bootstrap test (500 replicates) is shown next to the branches. The tree was drawn to scale, with branch lengths in the same units as those of the evolutionary distances used to infer the phylogenetic tree. The evolutionary distances were computed using the Maximum Composite Likelihood method and are in the units of the number of base substitutions per site. All positions containing gaps and missing data were eliminated from the dataset (Complete Deletion option). There were a total of 365 positions in the final dataset.

\section{Statistical analyses}

Participants were dichotomised into those who had or did not have detectable fungus in their sputum. We used the Chi-squared or Fisher's exact test to compare categorical variables and the t-test or Wilcoxon rank sum test to compare continuous variables (the latter for body mass index and employment duration, which were not normally distributed). We used ANOVA to test the overall difference in the distributions of sputum leukocyte cell types in fungal positive versus fungal negative samples. We assessed colinearity among the sputum cell type percentages for the entire study group using Pearson correlations. 
We used linear regression analysis to test the associations between selected independent variables (fungal status, and eosinophil and lymphocyte percentages) and forced midexpiratory flow (FEF25-75\%) $\%$ predicted as the dependent variable. All linear regression models also took into account the covariates age, smoking status (current versus former or never) and duration of employment. In addition to testing models in which only one of the independent predictors of interest was included along with the adjusting covariates, we also tested models including both fungal status and either eosinophil or lymphocyte percentage in the same model. We did so in order to assess the potential mediation of any fungal association with lung function by the presence of sputum eosinophils or lymphocytes. Because sputum eosinophil and lymphocyte percentages were strongly correlated with one another $(\mathrm{r}=0.72, \mathrm{p}<0.01)$ and both inversely correlated with the percent of macrophages $(r=-0.63$ and $r=-0.55$, respectively; both $\mathrm{p}<0.01$ ), we did not include any of these in multivariate models. Eosinophil and lymphocyte percentages, however, were only weakly negatively correlated with the percent neutrophils $(r=-0.22(\mathrm{p}<0.05)$ and $\mathrm{r}=-0.24(\mathrm{p}<0.10)$, respectively), allowing us to test whether inclusion of that variable had a substantive impact on the mediation by eosinophils of the association of fungal contamination with lung function. All analyses were performed using SPSS version 20 (IBM Corp., Armonk, NY, USA).

\section{Results}

Out of the targeted population of 79 males, we did not study those who did not provide consent $(n=7)$, did not include those ineligible based on current antibiotic use $(n=4)$ and excluded those without reproducible quality spirometry $(n=8)$ or who were unable to expectorate adequate sputum samples $(n=6)$. We ultimately analysed data for $54(68 \%)$ wood process workers employed at the four targeted worksites. Of those studied, 19 (35\%) produced a sputum sample in which fungus was detected, while 35 (65\%) were sputum fungus negative. In the genomic fungal characterisation, hits generated using standard search parameters indicated the fungus belonged to the genus Candida. A phylogenetic tree constructed from the hits of NCBI BLASTN search results is presented in figure 1. The sequence has been submitted to NCBI database and can be accessed through the accession number GenBank KF483576.1. Fungus was not detected in saliva sample stains or cultures (figure 1).

The characteristics of the participants by fungal status are presented in table 1. The fungus-positive group was, on average, 5.6 years older $(p=0.04)$ but there were no other statistically significant differences in height, weight, smoking status, employment duration or history of parental asthma.

\section{Respiratory symptoms}

The prevalence of reported respiratory symptoms based on questionnaire responses is presented in figure 2 . Although all of respiratory symptoms elicited were more prevalent among the participants with fungus-positive sputum, the differences were statistically significant only for phlegm, cough and haemoptysis $(\mathrm{p}<0.05)$. Although the latter was the least common complaint, it was reported by seven $(37 \%)$ participants in the fungus-positive group but only by two (6\%) among the others. Dermatological complaints, which we also ascertained as a possible adverse health effect of fungal contamination, were also was twice as common among the fungus-positive group (68.4\% versus $37.1 \%$; $\mathrm{p}=0.06)$ (figure 2 ).

\section{Lung function}

Lung function is presented in table 2. There were nonsignificant deficits of $60 \mathrm{~mL}$ in forced vital capacity (FVC) and $80 \mathrm{~mL}$ in forced expiratory volume in $1 \mathrm{~s}$ (FEV1) associated with fungal positive status. FEF 25-75\%, however, was significantly reduced in the fungus-positive group, both in absolute terms as and a percentage of the predicted value $(13 \%$ difference, $\mathrm{p}<0.01)$.

FIGURE 1 Phylogenetic tree for 11 taxa of Candida (see Methods section for further details).
gil242253910lemb|FM178339.1|Candida xyl gil166239761/gb|EU315758.1/Candida inco gi|388894631/gb|JQ726601.1|Candida inco gil539184692/gb|KF483576.1|Candida sp. gil166239760|gb|EU315757.1|Candida inco gil452056218|ref|NR077074.1|Candida xy gil349587278|gb|JF896575.1|Candida inco gil6333962092/gb|KJ546152.1 ICandida inco gil3888894634|gb|JQ726604.1|Candida xylo gil349587277|gb|JF896574.1|Candida xylo gil407196275|gb|JX169806.1|Candida sp. 


\section{TABLE 1 Characteristics of 54 wood mill worker study participants}

\section{Parameters}

Participants $\mathbf{n}$

Age years

Height $\mathrm{cm}$

BMI $\mathrm{kg} \cdot \mathrm{m}^{-2}$

Ever-smokers

Employment years

Parental asthma
Fungus positive

19

$42.5 \pm 10.4$

$172.05 \pm 4.76$

$21.9 \pm 3.3$

$8(42.1)$

$15.8 \pm 8.1$

6 (31.6)
Fungus negative

35

$36.9 \pm 5.2$

$173.20 \pm 5.42$

$21.2 \pm 2.3$

12 (34.3)

$13.0 \pm 4.2$

7 (20.0) p-value

0.04

0.43

0.44

0.79

0.18

0.54

Data are presented as mean \pm SD or $\mathrm{n}(\%)$, unless otherwise stated. All participants were male. $\mathrm{p}$-values are based on t-test for age and height, Wilcoxon rank sum test for body mass index (BMI) and employment years, and Chi-squared test for smoking status and parental asthma.

\section{Cellular distribution in sputum}

The distribution of sputum leukocytes by fungal status is presented in table 3 . The overall patterns of the proportions of eosinophils, lymphocytes, macrophages and neutrophils differed significantly between the two groups (ANOVA $\mathrm{p}<0.01$ ). The median eosinophil percentage in the sputum samples of the fungus-positive workers was almost three-fold higher than in the fungus-negative group $(9.25 \%$ versus $3.25 \%)$. The proportion of sputum lymphocytes was also higher in the in the fungus-positive workers compared with the fungus-negative (median $4.25 \%$ versus $1.75 \%$ ) while, conversely, the alveolar macrophage percentage was lower (22.5\% versus $31.25 \%)$. The median neutrophil percentage was similar between the two groups.

The associations between fungal detection, sputum eosinophil percentage and $\mathrm{FEF} 25-75 \%$ \% predicted are presented in table 4 . The presence of sputum fungus and eosinophils were each independently associated with reduced $\mathrm{FEF} 25-75 \% \%$ predicted, with the associated model explanatory power $\left(\mathrm{R}^{2}\right)$ ranging from 0.45 to 0.49 . When combined in the same model (model 3), the association of sputum fungus with reduced FEF25-75\% was markedly attenuated, while the relationship of eosinophil percentage to the dependent lung function variable was only modestly weakened, consistent with mediation of the observed fungal effect by eosinophil percentage. The addition of sputum macrophage percent to model 3 had little effect on the overall $R^{2}(0.48)$ and was not statistically associated with FEF25-75\% $(\beta=0.19,95 \%$ CI $-0.30-0.69)$. When fungal contamination and percentage lymphocytes were tested together, fungus remained associated with FEF25-75\% ( $\beta=-10.8,95 \%$ CI $-17.9--3.7)$ while the percentage lymphocytes did not $(\beta=-0.7,95 \%$ CI $-2.7-1.3$ ) (data not shown).

FIGURE 2 Prevalence of symptoms among the sawmill workers comparing those with and without fungus-positive sputum samples.

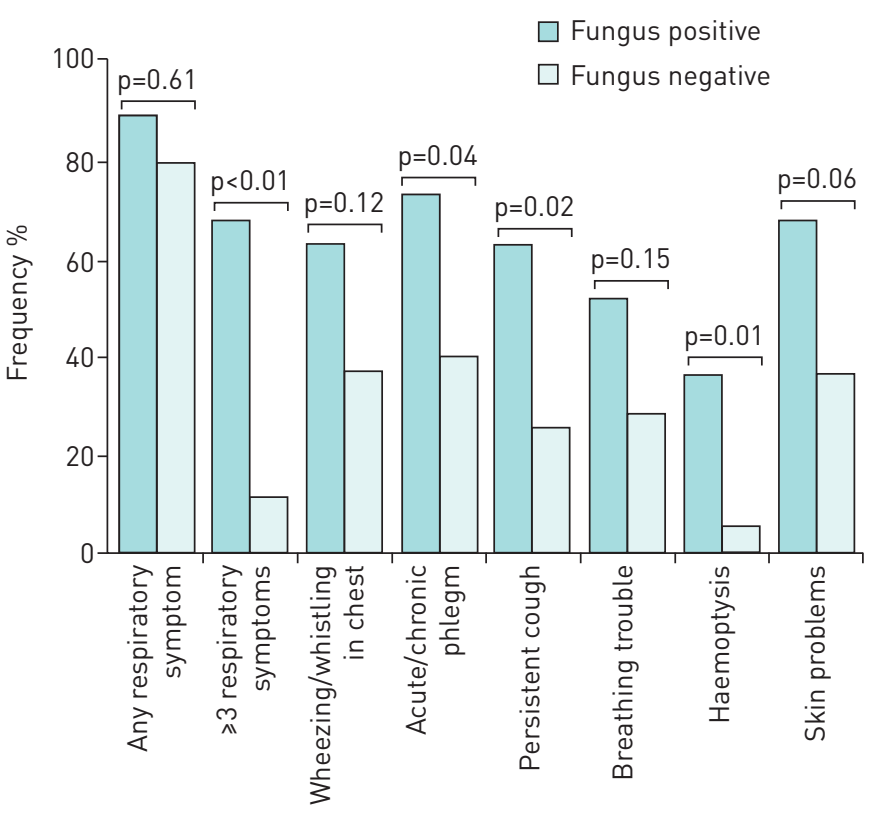




\begin{tabular}{|c|c|c|c|}
\hline Parameters & Fungus positive & Fungus negative & $p$-value \\
\hline Participants n & 19 & 35 & \\
\hline FVC L & $2.83 \pm 0.30$ & $2.89 \pm 0.24$ & 0.44 \\
\hline FVC $\%$ predicted & $79.0 \pm 8.2$ & $78.0 \pm 8.3$ & 0.70 \\
\hline FEV1 L & $2.12 \pm 0.29$ & $2.20 \pm 0.28$ & 0.36 \\
\hline FEV $1 \%$ predicted & $76.7 \pm 10.2$ & $75.2 \pm 11.2$ & 0.61 \\
\hline FEV $1 / F V C$ & $0.75 \pm 0.04$ & $0.76 \pm 0.06$ & 0.47 \\
\hline FEF $25-75 \%$ L.s ${ }^{-1}$ & $2.59 \pm 0.55$ & $2.98 \pm 0.47$ & 0.01 \\
\hline FEF25-75\% \% predicted & $69.2 \pm 9.95$ & $82.3 \pm 4.53$ & $<0.01$ \\
\hline
\end{tabular}

\section{Discussion}

Sawmill workers are occupationally exposed to a range of airborne contaminants. We detected fungal contamination by Candida sp. in the sputum samples of approximately one-third of the sawmill workers that we studied. Those sputum fungus-positive workers also had more frequent respiratory symptoms, increased sputum eosinophils and impaired lung function as measured by their flow at mid-lung volumes (FEF25-75\%). Moreover, fungal contamination with Candida was linked to the percentage of sputum eosinophils, with the latter appearing to mediate the observed association with lung function decrement.

Mould is commonly found in wood processing environments and is presumed to be a major risk factor in allergic responses that have been seen in workers in this industry $[1,21,23]$. Contamination of sputum by Candida sp., if present on repeated examination, is presumed to reflect airway contamination and this can be clinically relevant. The syndrome of allergic bronchopulmonary mycosis (ABPM), for example, even though most commonly associated with Aspergillus sp., is also seen with chronic airway candidiasis, and includes eosinophilia and airflow obstruction [34]. In the occupational milieu, chronic inhalational exposure to fungus is more closely linked to extrinsic allergic alveolitis than ABPM. Multiple occupational studies have quantified airborne fungal contamination in working environments in which various organic materials are processed, including wood products [6, 14-21]. Systematic assessment of fungal sputum contamination in occupational cohorts also has been studied but to a much more limited extent in selected high-risk cohorts other than woodworkers, such as malt, sugar cane and flax workers [35-37]. These studies have identified a high proportion of sputum samples positive for fungus although, unlike our study, the contamination was polymicrobial for a range of fungal species, and Candida sp., although present in 3-6\%, was not dominant in any of the cohorts [35,37]. An earlier study of a nonexposed occupational population (urban telephone company employees) found that a minority produced fungus-contaminated sputum samples and that Candida sp. also was uncommon among those [38].

The limitations in this study should be kept in view. We visually observed homogenous fungal spores (with lactophenol cotton blue stain) in sputum samples prior to culture. Nonetheless, the growth of Candida would have been promoted under the incubation conditions used (both by temperature and relatively short duration), which could have contributed to its dominance in the genotyping component of the analysis. Although we identified an association between sputum fungal contamination and both sputum eosinophilia and a lung function decrement, we cannot exclude the latter being a simply a marker

TABLE 3 Comparison of inflammatory cellular profile in the sputum between the study groups

\begin{tabular}{|c|c|c|c|c|}
\hline \multirow{2}{*}{$\begin{array}{l}\text { Sputum } \\
\text { cell type }\end{array}$} & \multicolumn{2}{|c|}{ Fungus positive" } & \multicolumn{2}{|c|}{ Fungus negative } \\
\hline & $\begin{array}{c}\text { Median } \\
\text { (interquartile range) }\end{array}$ & Range & $\begin{array}{c}\text { Median } \\
\text { (interquartile range) }\end{array}$ & Range \\
\hline Neutrophils \% & $62.75(58.0-67.25)$ & $52.25-69.5$ & $63.25(62.25-66.25)$ & $57.75-72.25$ \\
\hline Eosinophils \% & $9.25(8.5-10.25)$ & $6.75-11.5$ & $3.25(2.5-4.25)$ & $1.5-5.25$ \\
\hline Macrophages \% & 22.5 (18.75-28.5) & $16.75-33.75$ & 31.25 (29.5-32.625) & $24.0-35.5$ \\
\hline Lymphocytes \% & $4.25(3.75-4.875)$ & $3.5-7.5$ & $1.75(1.25-2.125)$ & $0.5-4.25$ \\
\hline
\end{tabular}


TABLE 4 Mediation analysis for the association between fungal appearance, lung function and sputum inflammatory cells

\begin{tabular}{lcccc} 
Model & Predictors & \multicolumn{3}{c}{ FEF25-75\% \% predicted } \\
\cline { 3 - 4 } & & $\boldsymbol{\beta}(\mathbf{9 5 \%} \mathbf{C I})$ & $\mathbf{p}$-value & $\mathbf{R}^{\mathbf{2}}$ \\
Model 1 & Fungus positivity & $-12.7(-16.9--8.5)$ & $<0.001$ & 0.45 \\
Model 2 & Eosinophil \% & $-2.1(-2.8--1.5)$ & $<0.001$ & 0.49 \\
Model 3 & Fungus positivity & $-3.5(-13.9-6.9)$ & 0.51 & 0.48 \\
& and eosinophil \% & $-1.6(-3.3-0.06)$ & 0.06 & \\
\hline
\end{tabular}

All models included age, smoking status (current versus former or never) and years of employment. FEF $25-75 \%$ : forced expiratory flow at $25-75 \%$ of forced vital capacity; $\beta$ : adjusted regression coefficient; $R^{2}$ : determination coefficient.

of another unmeasured and confounding coexposure. Such an exposure could be to other microbes, endotoxins, glucans or wood dust itself, none of which we measured. We did not have industrial hygiene sampling data or other direct exposure measures quantifying airborne concentrations of these exposures. Moreover, adequate characterisation of such exposures would have required multiple assessments at a number of different time-points given the likely variability in such bioaerosol exposures.

Despite observing a decrement in the spirometric measure of $\mathrm{FEF} 25-75 \%$, we did not detect an exposure-related decrement in FEV1 or the ratio of FEV1 to FVC, lung function variables of clinical relevance to asthma and chronic obstructive pulmonary disease (COPD). Radiographic imaging, which was not available, could have provided valuable additional data, for example, computed tomography-assessed air trapping or even the detection of fungus-associated changes that might have correlated with the sputum fungus-associated complaint of haemoptysis [39]. Although reduced FEF25-75\% indeed could be an early marker of airway disease that would later progress, our cross-sectional study cannot assess that prospective outcome. Furthermore, we did not have any clinical diagnostic assessment of the participants to assess the presence or absence of lung diseases such as asthma, COPD, bronchiectasis or tuberculosis.

Another study limitation is our lack of characterisation of the potential bacterial contents of the sputum, for example, by using bacterial ribosomal RNA sequencing or quantitative PCR techniques. We also acknowledge that we did not have access to a formal external control group of non-sawmill workers for sputum Candida testing and thus cannot comment on what the general population prevalence of such fungal detection might be. Rather, our analysis was more analogous to a nested design in which the Candida-positive workers were "cases" and those that were negative served as "referents" for comparison. Although we ultimately studied $68 \%$ of the target population, it is possible that those not studied differed in systematic ways that could have impacted the estimated prevalence of fungal detection. For example, spirometry test failure has been associated with airway disease [40]. In contrast, inability to produce sputum could theoretically be associated with an absence of disease, having the opposite association with fungal prevalence.

Because of these limitations, we cannot assume a causal association between the fungal contamination of the sawmill workers' sputum that we detected and airway inflammation or dysfunction. We can conclude, however, that contamination of the respiratory tract by Candida sp. in this study identified a group with a greater symptom burden, elevated sputum eosinophils and a decreased airflow at mid-lung volumes. These findings suggest that fungal contamination in this work environment may be linked to adverse health effects and that this question merits additional assessment, in particular, through targeted exposure assessment and longitudinal study of potential evolution in airflow deficits in those occupationally exposed in sawmills.

\section{References}

1 Wilhelmsson B, Jernudd Y, Ripe E, et al. Nasal hypersensitivity in wood furniture workers. An allergological and immunological investigation with special reference to mould and wood. Allergy 1984; 39: 586-595.

2 Hedenstierna G, Alexandersson R, Belin L, et al. Lung function and rhizopus antibodies in wood trimmers. A cross-sectional and longitudinal study. Int Arch Occup Environ Health 1986; 58: 167-177.

3 Dahlqvist $\mathrm{M}$, Johard U, Alexandersson R, et al. Lung function and precipitating antibodies in low exposed wood trimmers in Sweden. Am J Ind Med 1992; 21: 549-559.

4 Hessel PA, Herbert FA, Melenka LS, et al. Lung health in sawmill workers exposed to pine and spruce. Chest 1995; 108: 642-646.

5 Halpin DM, Graneek BJ, Turner-Warwick M, et al. Extrinsic allergic alveolitis and asthma in a sawmill worker: case report and review of the literature. Occup Environ Med 1994; 51: 160-164.

6 Mandryk J, Alwis KU, Hocking AD. Work-related symptoms and dose-response relationships for personal exposures and pulmonary function among woodworkers. Am J Ind Med 1999; 35: 481-490. 
7 Douwes J, McLean D, Slater T, et al. Asthma and other respiratory symptoms in New Zealand pine processing sawmill workers. Am J Ind Med 2001; 39: 608-615.

8 Jacobsen G, Schaumburg I, Sigsgaard T, et al. Non-malignant respiratory diseases and occupational exposure to wood dust. Part I. Fresh wood and mixed wood industry. Ann Agric Environ Med 2010; 17: 15-28.

9 Ljubičić Ćalušić A, Varnai VM, Cavlović AO, et al. Respiratory health and breath condensate acidity in sawmill workers. Int Arch Occup Environ Health 2013; 86: 815-825.

10 Deshpande A, Afshan A. Effect of chronic exposure of sawdust in workers employed in sawmills: a cross-sectional study. Sch J App Med Sci 2014; 2: 1202-1205.

11 Dutkiewicz J, Sorenson WG, Lewis DM, et al. Levels of bacteria, fungi and endotoxin in stored timber. Int Biodeterioration 1992; 30: 29-46.

12 Rask-Andersen A, Land CJ, Enlund $\mathrm{K}$, et al. Inhalation fever and respiratory symptoms in the trimming department of Swedish sawmills. Am J Ind Med 1994; 25: 65-67.

13 Alwis KU, Mandryk J, Hocking AD. Exposure to biohazards in wood dust: bacteria, fungi, endotoxins, and (1 $\rightarrow 3$ )-beta-D-glucans. Appl Occup Environ Hyg 1999; 14: 598-608.

14 Dennekamp M, Demers PA, Bartlett K, et al. Endotoxin exposure among softwood lumber mill workers in the Canadian province of British Columbia. Ann Agric Environ Med 1999; 6: 141-146.

15 Dutkiewicz J, Skórska C, Krysińska-Traczyk E, et al. Response of sawmill workers to work-related airborne allergens. Ann Agric Environ Med 2001; 8: 81-90.

16 Dutkiewicz J, Olenchock SA, Krysińska-Traczyk E, et al. Exposure to airborne microorganisms in fiberboard and chipboard factories. Ann Agric Environ Med 2001; 8: 191-199.

17 Krysińska-Traczyk E, Skórska C, Cholewa G, et al. Exposure to airborne microorganisms in furniture factories. Ann Agric Environ Med 2002; 9: 85-90.

18 Rusca S, Charrière N, Droz PO, et al. Effects of bioaerosol exposure on work-related symptoms among Swiss sawmill workers. Int Arch Occup Environ Health 2008; 81: 415-421.

19 Verma DK, Demers C, Shaw D, et al. Occupational Health and Safety Issues in Ontario Sawmills and Veneer/ Plywood Plants: A Pilot Study. J Environ Public Health 2010; 2010: 526487.

20 Gioffrè A, Marramao A, Iannò A. Airborne microorganisms, endotoxin, and dust concentration in wood factories in Italy. Ann Occup Hyg 2012; 56: 161-169.

21 Færden K, Lund MB, Mogens Aaløkken T, et al. Hypersensitivity pneumonitis in a cluster of sawmill workers: a 10-year follow-up of exposure, symptoms, and lung function. Int J Occup Environ Health 2014; 20: 167-173.

22 Guerrera E, Frusteri L, Giovinazzo R, et al. Presence of the microbiological risk in Umbrian sawmills. G Ital Med Lav Ergon 2006; 28: 466-471.

23 Veillette M, Cormier Y, Israël-Assayaq E, et al. Hypersensitivity pneumonitis in a hardwood processing plant related to heavy mold exposure. J Occup Environ Hyg 2006; 3: 301-307.

24 Moitra S, Blanc PD, Sahu S. Adverse respiratory effects associated with cadmium exposure in small-scale jewellery workshops in India. Thorax 2013; 68: 565-570.

25 Moitra S, Maity SG, Haldar P, et al. Trawler fuel exhaust and respiratory impairments: a cross-sectional pilot study among Indian fishermen working in informal sectors. Int J Occup Environ Health 2015; 21: 185-191.

26 Moitra S, Blanc PD, Brashier BB. Airflow obstruction among street vendors who refill cigarette lighters with liquefied petroleum gas. Int J Tuberc Lung Dis 2014; 18: 1126-1131.

27 Miller MR, Hankinson J, Brusasco V, et al. Standardisation of spirometry. Eur Respir J 2005; 26: 319-338.

28 Kamat SR, Tyagi NK, Rashid SSR. Lung function in Indian adult subjects. Lung India 1982; 1: 11-21.

29 Popov T, Gottschalk R, Kolendowicz R, et al. The evaluation of a cell dispersion method of sputum examination. Clin Exp Allergy 1994; 24: 778-783.

30 Moitra S, Chakraborty K, Bhattacharyya A, et al. Impact of occupational cadmium exposure on spirometry, sputum leukocyte count, and lung cell DNA damage among Indian goldsmiths. Am J Ind Med 2015; 58: 617-624

31 Saraiva-Romanholo BM, Barnabé V, Carvalho AL, et al. Comparison of three methods for differential cell count in induced sputum. Chest 2003; 124: 1060-1066.

32 Altschul SF, Gish W, Miller W, et al. Basic local alignment search tool. J Mol Biol 1990; 215: 403-410.

33 Tamura K, Dudley J, Nei M, et al. MEGA4: Molecular Evolutionary Genetics Analysis (MEGA) software version 4.0. Mol Biol Evol 2007; 24: 1596-1599.

34 Miyagawa $\mathrm{H}$, Yokota $\mathrm{S}$, Kajimoto $\mathrm{K}$, et al. A case report of pulmonary infiltration with eosinophilia syndrome induced by Candida albicans. Arerugi 1992; 41: 49-55.

35 Blyth W, Grant IWB, Blackadder ES, et al. Fungal antigens as a source of sensitization andrespiratory disease in Scottish maltworkers. Clin Allergy 1977; 7: 549-562.

36 Mehta SK, Sandhu RS. Immunological significance of Aspergillus fumigatus in Cane-sugar mills. Arch Environ Health 1983; 38: 41-46.

37 Ebeid N, Omar H, Refai M, et al. Respiratory mycotic affection: sputum examination of workers in a flax plant in Egypt. J Soc Occup Med 1982; 32: 66-72.

38 Comstock GW, Palmer CE, Stone RW, et al. Fungi in the sputum of normal men. Mycopathol Mycol Appl 1974; 54: 55-62.

39 Abel AT, Parwer S, Sanyal SC. Pulmonary mycetoma probably due to Candida albicans with complete resolution. Respir Med 1998; 92: 1079-1091.

40 Becklake MR. Epidemiology of spirometric test failure. Br J Ind Med 1990; 47: 73-74. 